\title{
Chunk-Based Energy Efficient Resource Allocation in OFDMA Systems
}

\author{
Yong Li, ${ }^{1}$ Zhengguang Zheng, ${ }^{2}$ Mingyue Zhao, ${ }^{3}$ Shan Jin, ${ }^{4}$ and Zhangqin Huang ${ }^{1}$ \\ ${ }^{1}$ The Institute of Embedded Software and Systems, Beijing University of Technology, Beijing 100022, China \\ ${ }^{2}$ Department of Vehicle Information Systems, North Information Control Group Co., Ltd., Guangzhou 510220, China \\ ${ }^{3}$ Department of Physical, Shunde High School, Foshan 528300, China \\ ${ }^{4}$ Department of National Key Laboratory of Science and Technology on Communications, \\ University of Electronic Science and Technology of China, Chengdu 610054, China
}

Correspondence should be addressed to Zhengguang Zheng; zhguangzheng@gmail.com

Received 22 August 2013; Revised 2 November 2013; Accepted 6 November 2013

Academic Editor: Zhan Shu

Copyright (C) 2013 Yong Li et al. This is an open access article distributed under the Creative Commons Attribution License, which permits unrestricted use, distribution, and reproduction in any medium, provided the original work is properly cited.

Energy efficiency (EE) capacity analysis of the chunk-based resource allocation is presented by considering the minimum spectrum efficiency (SE) constraint in downlink multiuser orthogonal frequency division multiplexing (OFDM) systems. Considering the minimum SE requirement, an optimization problem to maximize EE with limited transmit power is formulated over frequency selective channels. Based on this model, a low-complexity energy efficient resource allocation is proposed. The effects of system parameters, such as the average channel gain-to-noise ratio (CNR) and the number of subcarriers per chunk, are evaluated. Numerical results demonstrate the effectiveness of the proposed scheme for balancing the EE and SE.

\section{Introduction}

The unprecedented expansion of high data rate wireless networks has triggered steadily increase in energy consumption and left a significant environmental footprint. It is reported that the energy usage for radio access can account for up to more than 70 percent of the total energy bill for a mobile operator $[1,2]$. Thus, making information and communication technologies (ICT) applications greener can not only reduce the greenhouse gas emissions but also help operators attain long-term profitability.

Orthogonal frequency division multiplexing access (OFDMA) has become a promising technique due to its improved immunity to fast fading and flexibility in resource allocation [3]. Most of the research on resource allocation in OFDMA systems assumed single subcarrier allocation, where water-filling power distribution algorithm can be used [3-5]. However, the single-subcarrier-based resource allocation schemes need comparative more overhead and complicated implementation when employed with large number of subcarriers [6]. In order to reduce the overhead and complexity, the correlation between adjacent subcarriers should be considered. A set of contiguous subcarriers are grouped into one chunk and a chunk is regarded as the minimum unit for bandwidth allocation to users.

In previous works, chunk-based resource allocation schemes for OFDMA systems mainly intend to increase the system capacity or spectrum efficiency (SE) [6-8]. Reference [6] gives the optimal chunk allocation that maximizes a utility function of average user rates for a wireless OFDMA system under different power control policies. Reference [7] presents the performance analysis of the chunk-based allocation for downlink multiuser uncoded OFDMA systems with adaptive modulation. Reference [8] studies the joint chunk and power and bit allocation schemes to maximize the system capacity under a total transmit power constraint.

Besides the capacity improvement, the energy efficiency (EE) has been becoming equally or even more important than SE for green radio. Energy efficient design has received much attention from both industry and academia [1]. The EE optimization for single-subcarrier-based OFDMA systems has been investigated in [9-11]. Reference [9] addresses the 
energy efficient link adaptation to achieve the maximum EE for frequency-selective fading channels. Reference [10] studies the EE maximization subject to the minimum rate constraint. Reference [11] considers the EE optimization of OFDMA systems while ensuring the minimum rate requirement for each user. However, the single-subcarrier-based resource allocation is assumed in all of them, which incurs additional energy consumption due to its high implementation complexity.

The above discussions motivate us to investigate the EE optimization for chunk-based OFDMA systems. In this paper, we formulate an optimization problem to balance the tradeoff between SE and EE. The objective function is the EE which is measured by instantaneous "bits-per-Joule." The SE is assured by imposing a required minimum data rate into the optimization problem. This paper proposes a low-complexity algorithm to obtain high EE for each channel realization. Furthermore, for a fixed chunk assignment, an optimal power allocation with closed-form expression is also derived.

The rest of this paper is organized as follows. In Section 2, the system model and optimization objective are described. In Section 3, the chunk-based resource allocation scheme consisting of power allocation and chunk scheduling are derived. Section 4 provides numerical results. Finally, conclusions are drawn in Section 5.

\section{System Model}

Consider a single cellular downlink OFDMA system consisting of $K$ active users served by one base station. The system bandwidth $B$ is divided into $M$ chunks, each with $Q$ orthogonal narrowband subcarriers. Let $\mathscr{K}=\{1,2, \ldots, K\}$ and $\mathscr{M}=\{1,2, \ldots, M\}$ denote the sets of active users and chunks, respectively. In order to avoid interference among different users, one chunk is exclusively assigned to at most one user. Assume that each subcarrier is a Rayleigh fading channel which introduces a zero-mean circularly symmetric complex Gaussian noise.

The broadband channel is frequency selective and the normalized correlation coefficient between $n 1$ th subcarrier and $n 2$ th subcarrier of any one user can be written as [6]

$$
\rho_{n 1, n 2}=E\left\{h_{k, n 1}^{*} h_{k, n 2}\right\}=\frac{1}{\sqrt{1+\left((n 1-n 2) W / f_{c}\right)^{2}}}
$$

where $(\cdot)^{*}$ denotes complex conjugate, $f_{c}$ is the channel coherence bandwidth, and $W$ is the bandwidth of each subcarrier. In this paper, perfect instantaneous channel state information is assumed to be available at the base station. Let $h_{k, m, q}$ denote the frequency response for the $k$ th user at the $q$ th subcarrier of chunk $m$.

Let $\mathscr{M}_{k}$ be the index set of chunks assigned to user $k$. Denote $p_{k, m, q}$ as the transmit power of user $k$ on subcarrier $q$ of chunk $m$; then, the achievable data rate and total transmit power of user $k$ are

$$
\begin{gathered}
R_{k}=\sum_{m \in M_{k}} \sum_{q=1}^{Q} W f\left(p_{k, m, q}, g_{k, m, q}\right), \\
P_{k}=\sum_{m \in \mathscr{M}_{k}} \sum_{q=1}^{Q} p_{k, m, q},
\end{gathered}
$$

for $k \in \mathscr{K}$, where $g_{k, m, q}=\left|h_{k, m, q}\right|^{2} / \sigma^{2}$ is the channel gainto-noise, ratio (CNR), $\sigma^{2}$ is the power of additive Gaussian noise and $f\left(p_{k, m, q}, g_{k, m, q}\right)=\log _{2}\left(1+p_{k, m, q} g_{k, m, q}\right)$.

To guarantee the quality-of-service (QoS), the overall data rate $R$ should be restricted by

$$
R=\sum_{k=1}^{K} R_{k} \geq \check{R}
$$

where $\check{R}$ is the minimum rate requirement.

According to [11], EE is defined as the number of transmitted bits per unit energy consumption; thus, the EE can be given as

$$
U=\frac{R}{\alpha P+P_{c}},
$$

where $\alpha$ is the transmit power factor and the circuit power $P_{c}$ is

$$
P_{c}=P_{s}+\beta R
$$

where $P_{s}$ is the static circuit power and $\beta$ is the dynamic circuit power per unit data rate.

For a practical system, the total transmit power $P$ is limited, and we have

$$
P=\sum_{k=1}^{K} P_{k} \leq \widehat{P}
$$

where $\widehat{P}$ is the maximum allowable total transmit power.

Our objective is to optimize the chunk and power allocation in order to obtain the maximum EE under the required minimum rate constraint. Hence, the optimization problem can be formulated as

$$
\begin{array}{ll}
\max _{\mathscr{M}_{k} \in \mathscr{M}_{p, m, q}} U & =\frac{R}{\alpha P_{t}+P_{c}} \\
\text { s.t. } \quad \mathscr{M}_{k} \bigcap \mathscr{M}_{k^{\prime}}=\emptyset, \quad \forall k \neq k^{\prime}, \\
\\
\bigcup_{k=1}^{K} \mathscr{M}_{k} \subseteq \mathscr{M}, \\
P \leq \widehat{P}, \\
R \geq \check{R} .
\end{array}
$$




\section{Resource Allocation}

The optimization problem in ((7), (8), (9), (10), and (11)) is a mixed integer programming problem with nonlinear constraints. It is generally very hard to solve since the feasible set is not convex. Ideally, chunk and transmit power should be allocated jointly to achieve the optimal solution. There are $K^{M}$ possible chunk allocations with $K$ users and $M$ chunks. The maximum $\mathrm{EE}$ over all these chunk allocation schemes is the global maximum, and the corresponding chunk and power allocation is the optimal resource allocation scheme. However, the computational complexity is too high. In order to reduce complexity, we can divide the solving process into two steps: chunk allocation and power distribution. In this two-step method, we first consider chunk allocation then the power distribution. Section 3.1 describes a chunk allocation and the latter subsection presents the optimal power distribution for a fixed chunk allocation.

3.1. Chunk Allocation. For any given maximum total transmit power $\bar{P}$ without the required minimum rate constraint, the equivalent problem is a sum-rate maximization problem. Let us write the Lagrangian function

$$
\begin{aligned}
L\left(p_{k, m, q}, \lambda\right)= & \sum_{k=1}^{K} \sum_{m \in \mathscr{M}_{k}} \sum_{q \in m} f\left(p_{k, m, q}, g_{k, m, q}\right) \\
& +\lambda\left(\bar{P}-\sum_{k=1}^{K} \sum_{m \in \mathscr{M}_{k}} \sum_{q \in m} p_{k, m, q}\right),
\end{aligned}
$$

where $\lambda$ is the Lagrange multiplier.

The dual problem is defined as

$$
\theta=\min _{\lambda \geq 0} L(\lambda),
$$

where the dual objective is given by

$$
\theta(\lambda)=\max _{p_{k, m, q}} L\left(p_{k, m, q}, \lambda\right) .
$$

The optimal power distribution should maximize $\theta(\lambda)$, for all $k, m, q$, for all $\lambda$. More specifically speaking, the $k_{m}$ th user which maximize $\theta(\lambda)$ will be selected as the winner on chunk $m$ in this iteration

$$
k_{m}=\arg \max _{k \in \mathscr{K}} \sum_{q \in m}\left(f\left(p_{k, m, q}, g_{k, m, q}\right)-\lambda p_{k, m, q}\right) .
$$

The optimal transmit power for user $k$ on subcarrier $q$ in chunk $m$ is

$$
p_{k, m, q}= \begin{cases}\max \left(\mu-\frac{1}{g_{k, m, q}}, 0\right), & \text { if } k=k_{m} \\ 0, & \text { otherwise }\end{cases}
$$

where the power level is $\mu=1 / \lambda \ln 2$.

The core principle of the chunk allocation scheme is to assign the chunk with high capacity as much as possible for each user. Since both the throughput and the EE are nondecreasing with the number of subcarriers. In order to achieve high EE, we can set $\bar{P}=\widehat{P}$ and obtain the optimal chunk scheduling by iteratively exchanging information and updating the Lagrange multiplier. The goal of maximizing EE while ensuring a required minimum data rate is achieved by the power distribution in the next subsection.

3.2. Power Distribution. According to the above discussion, the data rate is maximized when the power is distributed among the subcarriers using water-filling algorithm. For a given chunk allocation, the optimal power distribution and system throughput continuously change as the power level varies. Then, the optimization problem can be reformulated as

$$
\begin{array}{rl}
\max _{p_{k, m, q}} & U=\frac{R}{\alpha P_{t}+P_{c}} \\
\text { s.t. } & P \leq \widehat{P}, \\
& R \geq \check{R} .
\end{array}
$$

It can be seen that the system data rate $R$ is a strictly increasing function of power level $\mu$ for $\mu \geq 0$. Let $\left\{g_{n}\right\}_{n=1}^{N}$, $(N=M Q)$ be the sequence of $\left\{g_{k_{m}, m, q}\right\}$, for all $m, q$, in descending order and let $p_{n}$ be the corresponding power.

Lemma 1. The minimum power level $\check{\mu}$ that satisfies $R=\check{R}$ is

$$
\check{\mu}=\min _{1 \leq I \leq N} 2^{\check{R} / I W}\left(\prod_{n=1}^{I} g_{n}\right)^{-(1 / I)} .
$$

Proof. Please see Appendix A.

Lemma 2. The maximum power level $\widehat{\mu}$ to maximize system rate under the total transmit power $\widehat{P}$ constraint is

$$
\widehat{\mu}=\min _{1 \leq I \leq N} \frac{1}{I}\left(\widehat{P}+\sum_{n=1}^{I} \frac{1}{g_{n}}\right) .
$$

Proof. Please see Appendix B.

Since we assume that the required minimum data rate can be achieved under the constraint (6), then $\check{\mu} \leq \widehat{\mu}$ and the problem ((17), (18), and (19)) can be rewritten as

$$
\begin{aligned}
& \max _{\mu} \quad U=\frac{R}{\alpha P_{t}+P_{c}} \\
& \text { s.t. } \check{\mu} \leq \mu \leq \widehat{\mu} .
\end{aligned}
$$

The primal variable of problem ((22), (23)) is one dimension, and the direct way to solve this problem is the brute search method over the region $[\check{\mu}, \widehat{\mu}]$. However, brute search has high complexity to achieve exact solution which poses a prohibitive computational burden at the base station. In order to solve this problem efficiently, we can further analyze the relationship between $\mathrm{EE}$ and power level. 

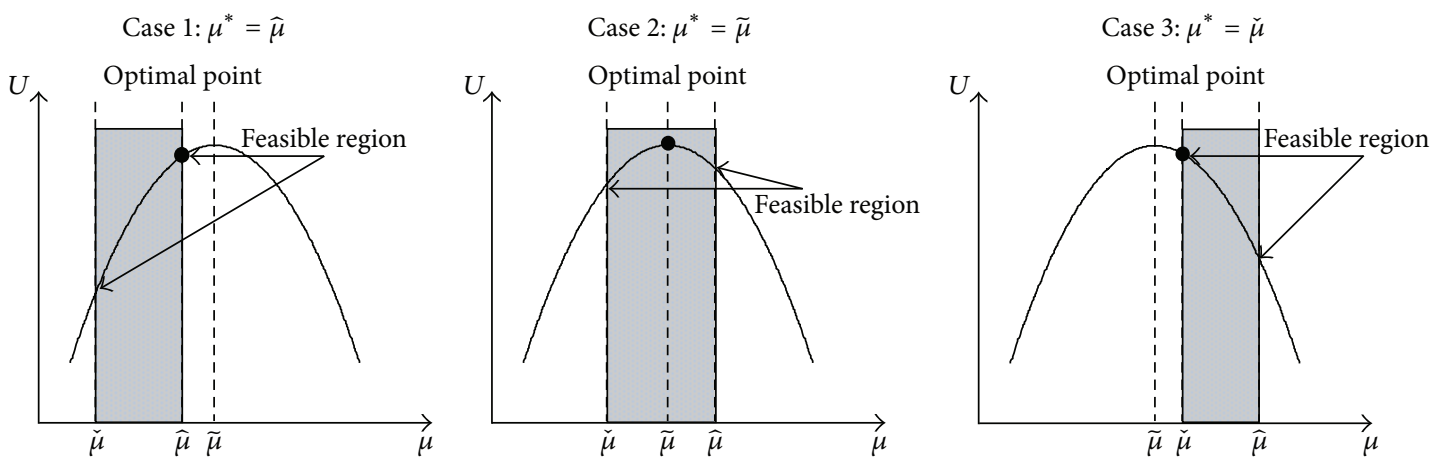

FIgURE 1: EE-power level relation in downlink OFDMA.

Lemma 3. To a certain determined chunk allocation, the EE is a quasiconcave function of power level $\mu$.

Proof. Please see Appendix C.

According to Lemma 3, EE $U$ is a quasiconcave function of power level $\mu$; hence, the optimal unconstrained power level always exists. Differentiating (22) with respect to $\mu$ and setting the derivative to zero, we can obtain

$$
\begin{aligned}
\frac{\partial U}{\partial \mu} & =F\left(1+\frac{P_{s}}{\alpha \widetilde{N} \mu}-\frac{G_{1}}{\mu}-\ln \left(\mu G_{2}\right)\right) \\
& =0
\end{aligned}
$$

or

$$
\frac{P_{s} G_{2}}{\alpha \widetilde{N} e}-\frac{G_{1} G_{2}}{e}=\frac{\mu G_{2}}{e} \ln \left(\frac{\mu G_{2}}{e}\right),
$$

where $e$ is the base of the natural logarithm, $F$ is a positive variable, $\widetilde{N}$ is the number of subcarriers with transmit power $p_{n}>0, G_{1}$, and $G_{2}$ are defined as

$$
\begin{gathered}
G_{1}=\frac{1}{\widetilde{N}} \sum_{n=1}^{\widetilde{N}} \frac{1}{g_{n}}, \\
G_{2}=\left(\prod_{n=1}^{\widetilde{N}} g_{n}\right)^{1 / \widetilde{N}},
\end{gathered}
$$

respectively. Let $X=\ln \left(\mu G_{2} / e\right)$ and let $Y=\left(P_{s} G_{2} / \alpha \widetilde{N} e\right)-$ $\left(G_{1} G_{2} / e\right)$; then, (25) can be expressed as

$$
X e^{X}=Y \text {. }
$$

Its solution is $X=W_{0}(Y)$, where $W_{0}(\cdot)$ denotes the real branch of the Lambert function [12]. Substituting this solution into (27), the optimal unconstrained power level $\tilde{\mu}$ without any constraint is

$$
\tilde{\mu}=\frac{e}{G_{2}} \exp \left[W_{0}\left(\frac{P_{s} G_{2}}{\alpha \widetilde{N} e}-\frac{G_{1} G_{2}}{e}\right)\right] .
$$

Figure 1 illustrates three possible curves of the relation between EE and power level that satisfy all constraints. So the optimal power level of the problem ((17), (18), and (19)) is

$$
\mu^{*}=\min (\max (\widetilde{\mu}, \check{\mu}), \widehat{\mu}) \text {. }
$$

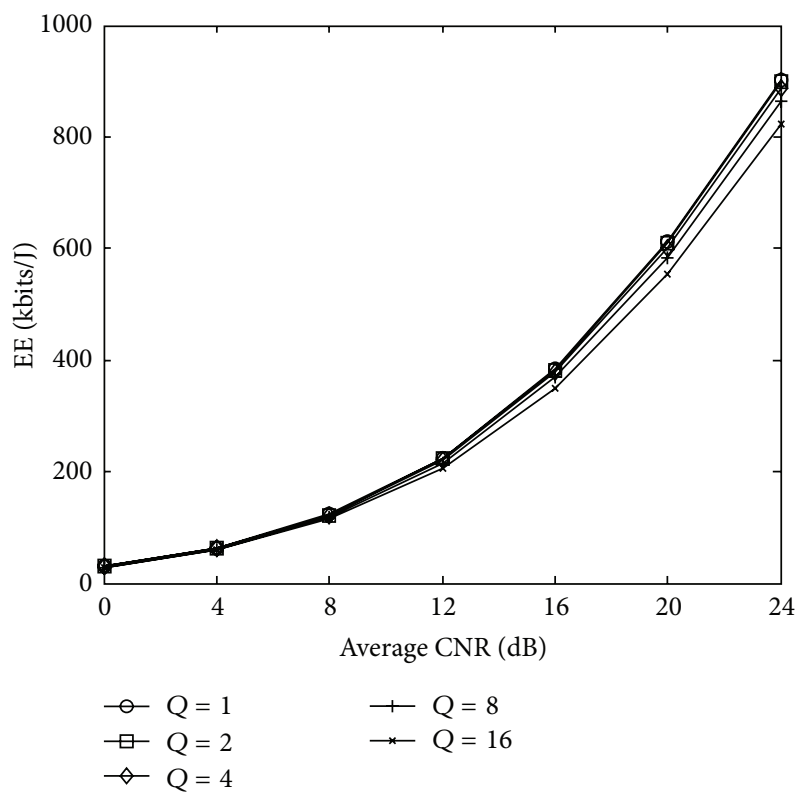

Figure 2: System EE versus average CNR in the case that $P_{s}=15 \mathrm{w}$ and $\beta=0$.

\section{Numerical Results}

In this section, we provide some simulation results to verify the effectiveness of the algorithm proposed in the previous sections. The system parameters are assumed as follows. The number of active users is 6 and the system bandwidth is divided into 256 subcarriers, each with bandwidth $W=$ $40 \mathrm{kHz}$. The channel is frequency selective and independent for all users. Assume that the correlation bandwidth between any two subcarriers of one user is the same for all users and $f_{c}=200 \mathrm{kHz}$. The Rayleigh fading channel can be generated based on the method in $[6,13]$. For sake of simplicity, all users have an identical average CNR and the transmit power factor, $\alpha$, is assumed to be 3 .

Figure 2 shows the performance of EE among the different sizes of each chunk. It can be seen, from Figures 2 and 3, that both EE and SE increase with the average CNR. This is because that energy efficient design tends to use less 


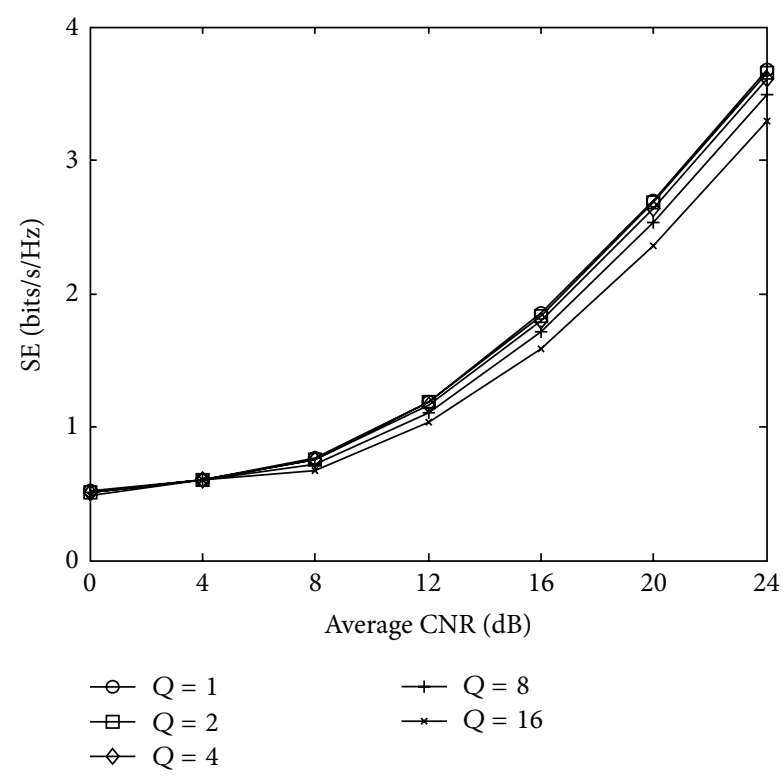

FIgURE 3: System SE versus average CNR in the case that $P_{s}=15 \mathrm{w}$ and $\beta=0$.

transmit power when CNR increases. The single-subcarrierbased resource allocation yields the highest system EE and SE. The gap in terms of EE or SE between $Q=16$ and $Q=8$ is significant. The gap between $Q=8$ and $Q=4$ becomes smaller, and the gap between $Q=1$ and $Q=2$ is even negligible. The chunk-based resource allocation has almost the same performance if the size of the chunk is smaller than the coherence bandwidth. The reason is that when the size of chunk decreases, the autocorrelation value among the channels of subcarriers within a chunk increases.

Figure 4 compares and indicates the influence of minimum SE requirement on the relation between $\mathrm{EE}$ and SE with different static circuit power and dynamic circuit power factors. Figure 5 shows the SE performance corresponding to the curves in Figure 4. From there, the EE decreases with the circuit power while the SE increases with the static circuit power and is independent of dynamic circuit power factor. From the curves of EE-versus- $\check{R}$, when the minimum rate requirement is smaller or larger than a certain threshold, energy efficient design leads to same performance. The reason is that in the lower $\check{R}$ regime, the energy-efficient transmission strategy is to operate exactly at the optimal unconstrained point which means $\mu^{*}=\tilde{\mu}$. When $\check{R}$ goes beyond the threshold, EE will decrease approximately linearly with $\check{R}$ because the transmit power dominates the total power consumption and data rate is logarithmic in transmit power. However, when SE is large enough, the EE is flat again. This means that the system has to work with maximum transmit power to reach the required minimum data rate and $\mu^{*}=\widehat{\mu}$.

\section{Conclusion}

In this paper, the EE-SE relation in a single cell downlink OFDMA system is investigated and the performance analysis

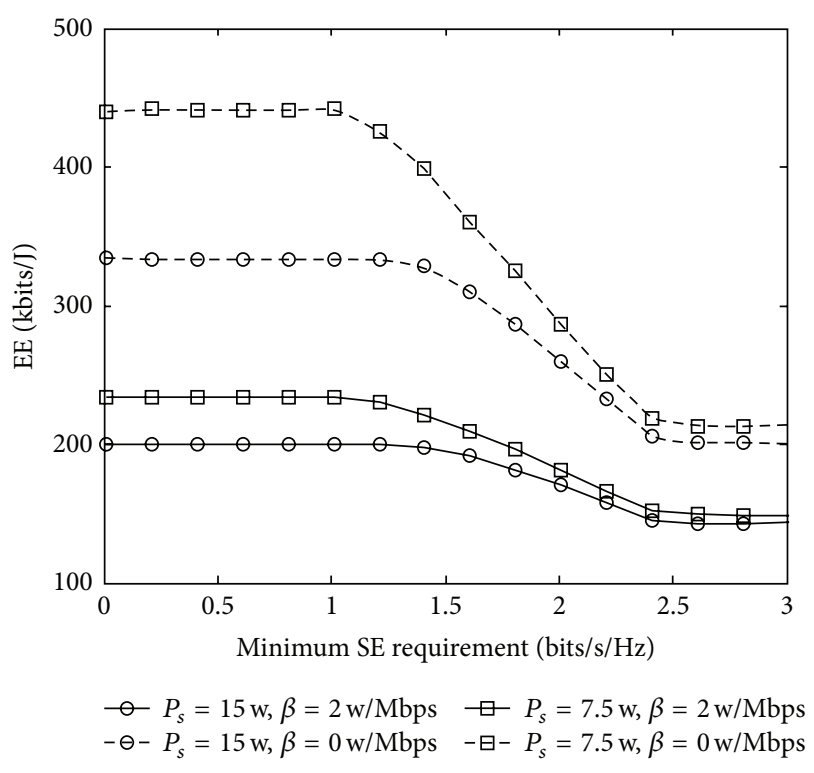

FIgURE 4: System EE versus average CNR in the case that CNR = $15 \mathrm{~dB}$.

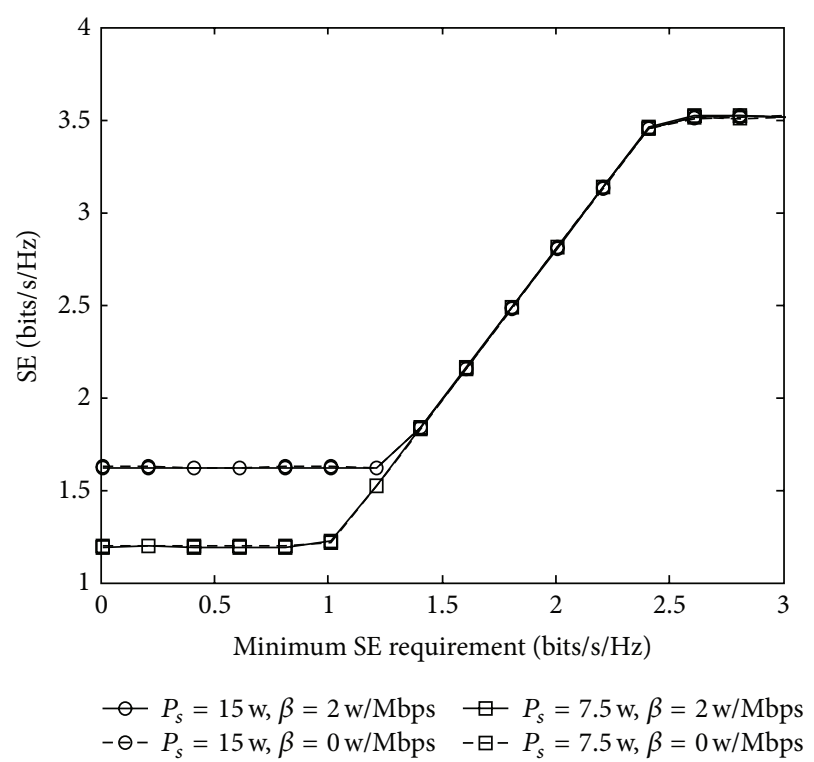

FIGURE 5: System SE versus average CNR in the case that CNR = $15 \mathrm{~dB}$.

of the chunk-based resource allocation is presented with energy-efficient design under the constraints on the minimum SE requirement and total available power. When the number of subcarriers per chunk is given, both EE and SE increase when the coherence bandwidth increase from a very small value. The performance of chunk-based resource allocation is very close to that of single-subcarrier-based allocation scheme when the bandwidth of each chunk is smaller than coherence bandwidth.

Simulation results verify the effectiveness of the proposed scheme in practical and demonstrate that it is much more suitable to green communications. Furthermore, the impact 
of the number of users and minimum SE requirement to the EE are also analyzed.

\section{Appendices}

\section{A. Appendix A}

Proof of Lemma 1. Let $\check{\mu}$ be the minimum power level corresponding to the minimum rate requirement $\check{R}$. We have

$$
\begin{aligned}
\check{R} & =W \sum_{n=1}^{N} \log _{2}\left(1+g_{n} p_{n}\right) \\
& =W \sum_{n=1}^{I} \log _{2}\left(1+\left(\check{\mu}-\frac{1}{g_{n}}\right) g_{n}\right) \\
& =W \log _{2}\left(\check{\mu}^{I} \prod_{n=1}^{I} g_{n}\right)
\end{aligned}
$$

or

$$
\check{\mu}=2^{\check{R} / W I}\left(\prod_{n=1}^{I} g_{n}\right)^{-1 / I},
$$

where $I \leq N, p_{n}=\check{\mu}-\left(1 / g_{n}\right)>0, n=1,2, \ldots, I$, and $\check{\mu} \leq$ $\left(1 / g_{n}\right), n=I+1, \ldots, N$.

For arbitrary $1 \leq I_{1}<I<I_{2} \leq N$, we have $\mu_{i}=$ $2^{\check{R} / W I_{i}}\left(\prod_{n=1}^{I_{i}} g_{n}\right)^{-1 / I_{i}}, i=1,2$. It can be seen that $\check{\mu} g_{n}>1$, $n=1, \ldots, I$, and $\check{\mu} g_{n} \leq 1, n=I+1, \ldots, N$. We derive the results that $\mu_{1}>\check{\mu}$ and $\mu_{2}>\check{\mu}$ as follows:

$$
\begin{aligned}
\frac{\mu_{1}}{\check{\mu}} & =\frac{2^{\check{R} / W I_{1}}\left(\prod_{n=1}^{I_{1}} g_{n}\right)^{-1 / I_{1}}}{\check{\mu}} \\
& =\frac{\left(\check{\mu}^{I} \prod_{n=1}^{I} g_{n} \prod_{n=1}^{I_{1}}\left(1 / g_{n}\right)\right)^{1 / I_{1}}}{\check{\mu}} \\
& =\left(\prod_{n=I_{1}+1}^{I} \check{\mu} g_{n}\right)^{1 / I_{1}} \\
& >1, \\
\frac{\mu_{2}}{\check{\mu}} & =\frac{2^{\check{R} / W I_{2}}\left(\prod_{n=1}^{I_{2}} g_{n}\right)^{-1 / I_{2}}}{\check{\mu}} \\
& =\frac{\left(\check{\mu} \prod_{n=1}^{I} g_{n} \prod_{n=1}^{I_{2}}\left(1 / g_{n}\right)\right)^{1 / I_{2}}}{\check{\mu}} \\
& \geq 1 . \\
& \left.\prod_{n=I+1}^{I_{2}} \frac{1}{\check{\mu} g_{n}}\right)^{1 / I_{2}}
\end{aligned}
$$

Since $\mu_{i}>0(i=1,2)$, the optimal power level $\check{\mu}$ that satisfies $R=\check{R}$ is

$$
\check{\mu}=\min _{1 \leq I \leq N} 2^{\check{R} / W I}\left(\prod_{n=1}^{I} g_{n}\right)^{-1 / I} .
$$

\section{B. Appendix B}

Proof of Lemma 2. For a given allowable transmit power $\widehat{P}$, the optimal power allocation can be achieved by classical water-filling method. The true power level is assumed to be $\widehat{\mu}$. We have

$$
\widehat{P}=\sum_{n=1}^{N} p_{n}=\sum_{n=1}^{I}\left(\widehat{\mu}-\frac{1}{g_{n}}\right)=I \widehat{\mu}-\sum_{n=1}^{I} \frac{1}{g_{n}}
$$

or

$$
\widehat{\mu}=\frac{1}{I}\left(\widehat{P}+\sum_{n=1}^{I} \frac{1}{g_{n}}\right),
$$

where $I \leq N, p_{n}=\widehat{\mu}-\left(1 / g_{n}\right)>0, n=1,2, \ldots, I$, and $\widehat{\mu} \leq$ $1 / g_{n}, n=I+1, \ldots, N$.

For arbitrary $1 \leq I_{1}<I<I_{2} \leq N$, we have $\mu_{i}=$ $\left(1 / I_{i}\right)\left(\widehat{P}+\sum_{n=1}^{I_{i}}\left(1 / g_{n}\right)\right), i=1,2$. Because $\widehat{\mu}>1 / g_{n}, n=$ $1, \ldots, I$, and $\widehat{\mu} \leq 1 / g_{n}, n=I+1, \ldots, N$, in (B.2), we derive the results that $\mu_{1}>\widehat{\mu}$ and $\mu_{2}>\widehat{\mu}$ as follows:

$$
\begin{aligned}
\mu_{1}-\widehat{\mu} & =\frac{1}{I_{1}}\left(I \widehat{\mu}-\sum_{n=1}^{I} \frac{1}{g_{n}}+\sum_{n=1}^{I_{1}} \frac{1}{g_{n}}\right)-\widehat{\mu} \\
& =\frac{1}{I_{1}}\left[\left(I-I_{1}\right) \widehat{\mu}-\sum_{n=I_{1}+1}^{I} \frac{1}{g_{n}}\right] \\
& =\frac{1}{I_{1}} \sum_{n=I_{1}+1}^{I}\left(\widehat{\mu}-\frac{1}{g_{n}}\right) \\
& >0, \\
\mu_{2}-\widehat{\mu} & =\frac{1}{I_{2}}\left(I \widehat{\mu}-\sum_{n=1}^{I} \frac{1}{g_{n}}+\sum_{n=1}^{I_{2}} \frac{1}{g_{n}}\right)-\widehat{\mu} \\
& =\frac{1}{I_{2}}\left[-\left(I_{2}-I\right) \widehat{\mu}+\sum_{n=I+1}^{I_{2}} \frac{1}{g_{n}}\right] \\
& =-\frac{1}{I_{2}} \sum_{n=I+1}^{I_{2}}\left(\widehat{\mu}-\frac{1}{g_{n}}\right) \\
& \geq 0 .
\end{aligned}
$$

Hence, the true power level $\widehat{\mu}$ corresponding to the maximum allowable transmit power is

$$
\widehat{\mu}=\min _{1 \leq I \leq N} \frac{1}{I}\left(\widehat{P}+\sum_{n=1}^{I} \frac{1}{g_{n}}\right) .
$$

Here, Lemma 2 is proved. 


\section{Appendix C}

Proof of Lemma 3. From problem (7), the objective of EE optimization is modeled as

$$
\max _{p_{n}} U(P)=\frac{1}{\left(\left(\alpha P+P_{s}\right) / R(P)\right)+\beta},
$$

where $P$ is the total transmit power and $R(p)$ is the corresponding data rate.

Since $\alpha>0, R>0, P>0$, and $P_{s}>0$, the EE $U$ is a strictly monotone decreasing function of $\left(\alpha P+P_{s}\right) / R$. Then, the equivalent optimization problem is reformulated as

$$
\max _{p_{n}} \bar{U}(P)=\frac{R(P)}{\alpha P+P_{s}} .
$$

For arbitrary $\theta \in(0,1)$, since $R(P)$ is concave function of $P$, we have

$$
\begin{aligned}
\bar{U}\left(\theta x_{1}+(1-\theta) x_{2}\right) & \geq \frac{\theta R\left(x_{1}\right)+(1-\theta) R\left(x_{2}\right)}{\theta\left(\alpha x_{1}+P_{s}\right)+(1-\theta)\left(\alpha x_{2}+P_{s}\right)} \\
& \geq \min \left(\bar{U}\left(x_{1}\right), \bar{U}\left(x_{2}\right)\right) .
\end{aligned}
$$

According to [14], we can conclude that $U$ is quasiconcave for $P \geq 0$, since $P$ is a monotonic increasing function of water level for $\mu>1 / \max g_{n}$. Hence, EE is a quasiconcave function of water level.

\section{Acknowledgment}

This work is partially supported by the Beijing Municipal Natural Science Foundation (no. 4122010).

\section{References}

[1] Y. Chen, S. Zhang, S. Xu, and G. Y. Li, "Fundamental trade-offs on green wireless networks," IEEE Communications Magazine, vol. 49, no. 6, pp. 30-37, 2011.

[2] Z. Hasan, H. Boostanimehr, and V. K. Bhargava, "Green cellular networks: a survey, some research issues and challenges," IEEE Communications Surveys and Tutorials, vol. 13, no. 4, pp. 524540, 2011.

[3] W. W. L. Ho and Y.-C. Liang, "Optimal resource allocation for multiuser MIMO-OFDM systems with user rate constraints," IEEE Transactions on Vehicular Technology, vol. 58, no. 3, pp. 1190-1203, 2009.

[4] K. Seong, M. Mohseni, and J. M. Cioffi, "Optimal resource allocation for OFDMA downlink systems," in Proceedings of the IEEE International Symposium on Information Theory (ISIT '06), pp. 1394-1398, July 2006.

[5] Y.-B. Lin, T.-H. Chiu, and Y. T. Su, "Optimal and near-optimal resource allocation algorithms for OFDMA networks," IEEE Transactions on Wireless Communications, vol. 8, no. 8, pp. 4066-4077, 2009.

[6] N. Gao and X. Wang, "Optimal subcarrier-chunk scheduling for wireless OFDMA systems," IEEE Transactions on Wireless Communications, vol. 10, no. 7, pp. 2116-2123, 2011.
[7] H. Zhu and J. Wang, "Chunk-based resource allocation in OFDMA systems-part I: chunk allocation," IEEE Transactions on Communications, vol. 57, no. 9, pp. 2734-2744, 2009.

[8] H. Zhu and J. Wang, "Chunk-based resource allocation in OFDMA systems-part II: joint chunk, power and bit allocation," IEEE Transactions on Communications, vol. 60, no. 2, pp. 499-509, 2012.

[9] G. Miao, N. Himayat, and G. Y. Li, "Energy-efficient link adaptation in frequency-selective channels," IEEE Transactions on Communications, vol. 58, no. 2, pp. 545-554, 2010.

[10] Z. Chong and E. Jorswieck, "Analytical foundation for energy efficiency optimisation in cellular networks with elastic traffic," in Proceedings of the 3rd International ICST Conference on Mobile Lightweight Wireless Systems, 2011.

[11] C. Xiong, G. Y. Li, S. Zhang, Y. Chen, and S. Xu, "Energy- and spectral-efficiency tradeoff in downlink OFDMA networks," IEEE Transactions on Wireless Communications, vol. 10, no. 11, pp. 3874-3886, 2011.

[12] R. M. Corless, G. H. Gonnet, D. E. G. Hare, D. J. Jeffrey, and D. E. Knuth, "On the Lambert W function," Advances in Computational Mathematics, vol. 5, no. 4, pp. 329-359, 1996.

[13] B. Natarajan, C. R. Nassar, and V. Chandrasekhar, "Generation of correlated Rayleigh fading envelopes for spread spectrum applications," IEEE Communications Letters, vol. 4, no. 1, pp. 9$11,2000$.

[14] S. Boyd and L. Vandenberghe, Convex Optimization, Cambridge University Press, Cambridge, Mass, USA, 2004. 


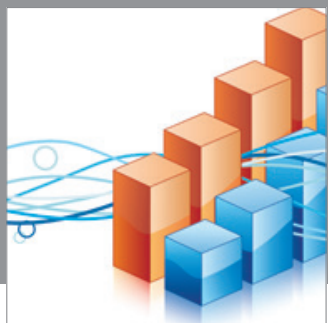

Advances in

Operations Research

mansans

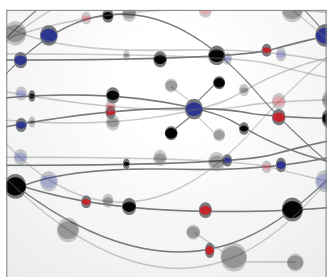

The Scientific World Journal
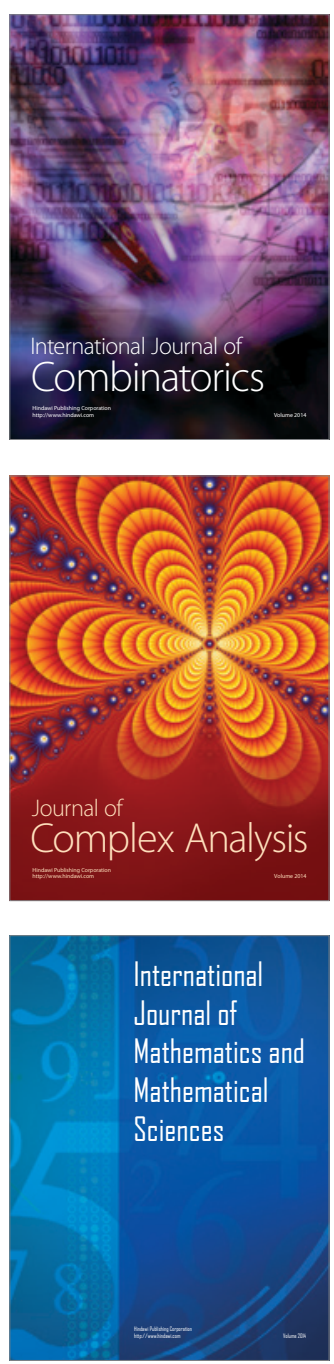
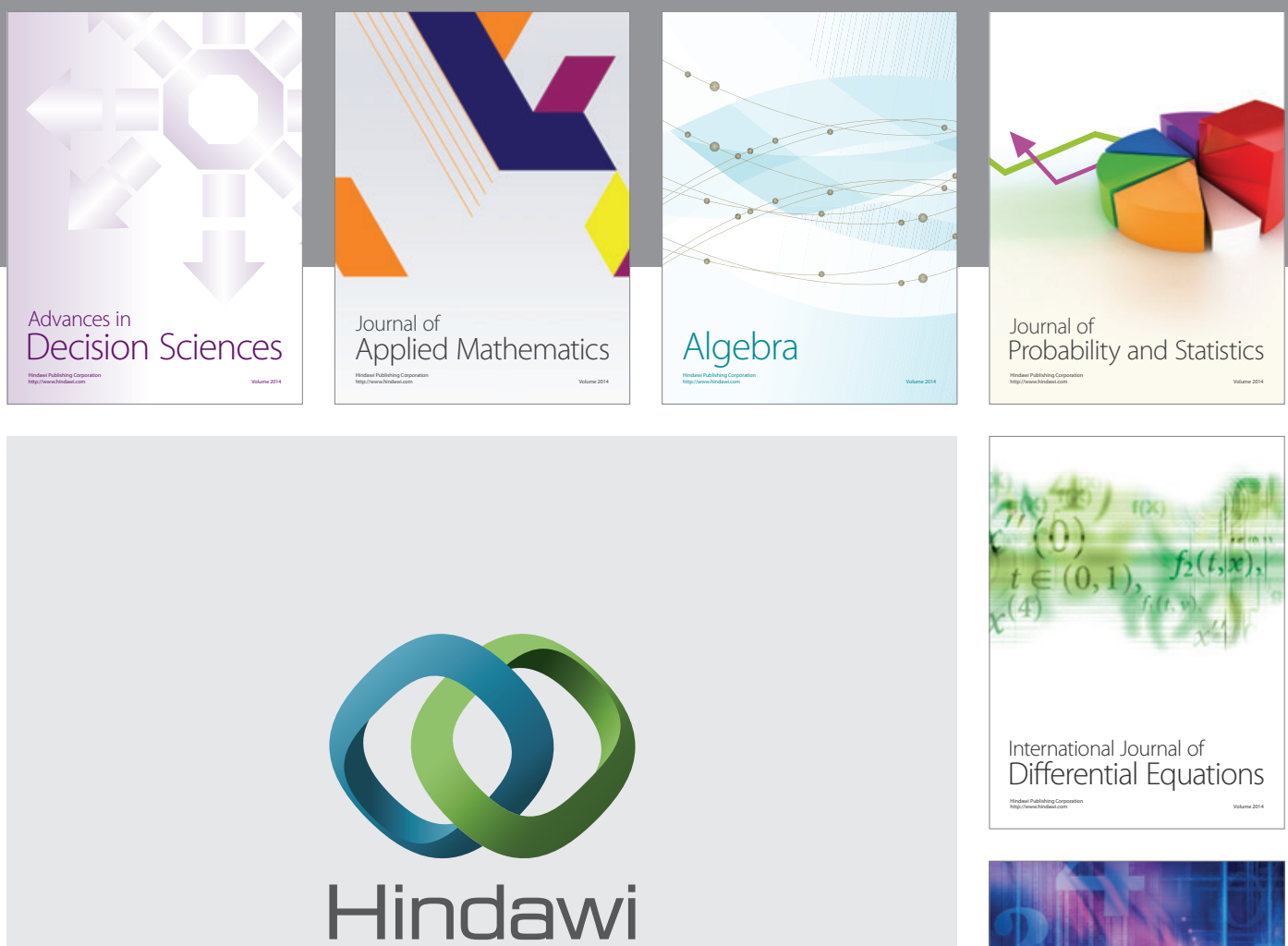

Submit your manuscripts at http://www.hindawi.com
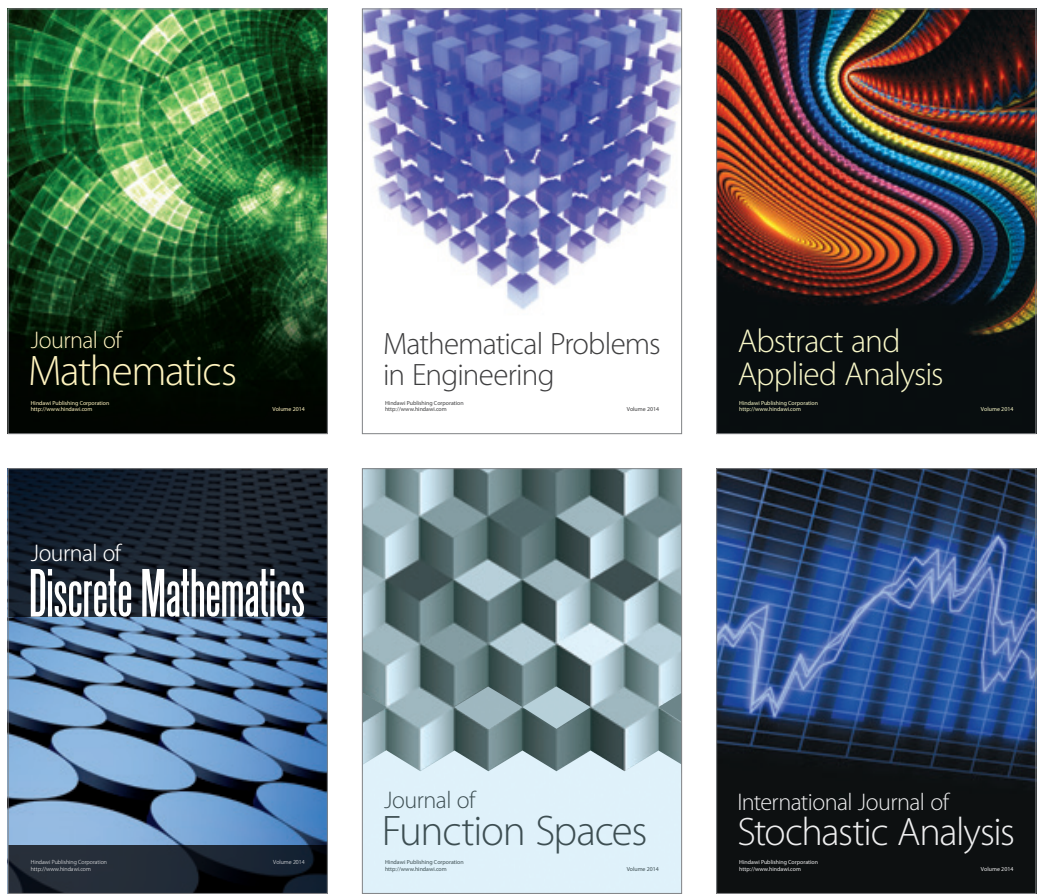

Journal of

Function Spaces

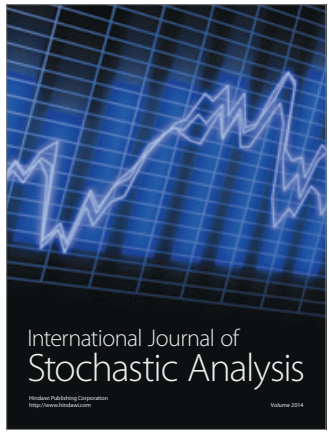

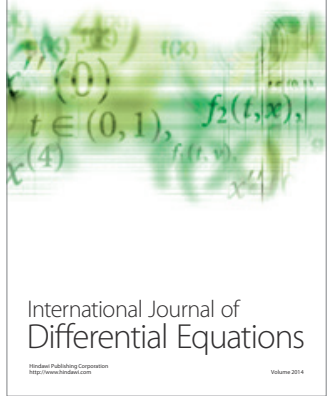
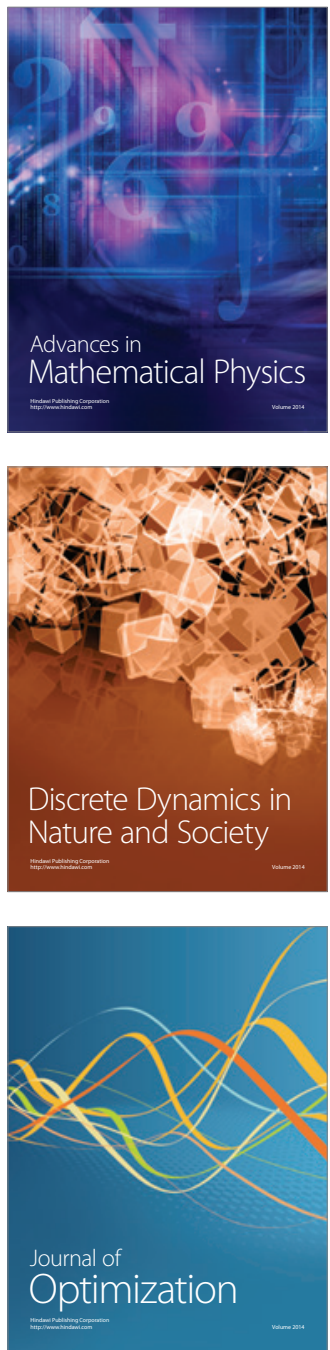